\title{
Th17 cell differentiation induced by cytopathogenic biotype BVDV-2 in bovine PBLCs
}

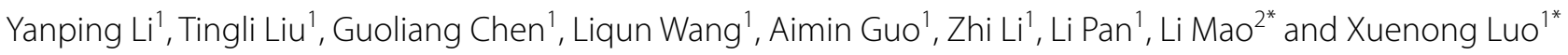

\begin{abstract}
Background: Bovine viral diarrhea virus (BVDV) is a major pathogen that causes bovine viral diarrhea/mucosal disease (BVD-MD), which has become a global infectious disease due to its wide spread and the lack of effective treatment. The process of BVDV infection is complex. Once infected, host immune cells are activated and modulated. As a major immune cell, peripheral blood lymphocyte cells (PBLCs) are the primary target of BVDV. In order to further understand the mechanism of BVDV- host interaction, the expression profiles of host lymphocytes mRNAs associated with BVDV infection were investigated by transcriptomic sequencing analysis.

Results: The transcriptomic sequencing analysis was performed on bovine PBLCs infected with CP BVDV-2 GS2018 after $12 \mathrm{~h}$ of infection. Gene expression profiling demonstrated that 1052 genes were differentially expressed in GS2018 infected PBLCs compared with the control group. Of these genes, 485 genes were up-regulated and 567 were down-regulated. The 19 differential expressed genes (DEGs) were selected for validation using quantitative real-time PCR and the results were consistent with the results of RNA-Seq. Gene ontology enrichment and KEGG pathway analysis showed that 1052 DEGs were significantly enriched in 16 pathways, including cytokine-cytokine receptor interaction, IL17, PI3K-Akt, MAPK and TNF signaling pathway. PPI network analysis showed that IL17A, IFN- $\gamma$ and TNF-a interacted with various proteins and may play crucial roles in BVDV-2 infection. Of note, we confirmed that GS2018 induced Th17 cell differentiation in PBLCs and persistently increased the expression levels of IL17A. In turn, the replication of GS2018 was inhibited by IL17A.
\end{abstract}

Conclusion: In this study, the transcription changes of DEGs related to host immune responses in bovine PBLCs were caused by CP BVDV-2 infection. In particular, the effector molecules IL17A of Th17 cells were significantly up-regulated, which inhibited viral replication. These results will contribute to exploration and further understanding of the host immune response mechanism and interaction between host and BVDV-2.

Keywords: BVDV-2, RNA-Seq, Th17 cell differentiation, Viral replication, Immune response

*Correspondence: mao-li@live.cn; luoxuenong@caas.cn

${ }^{1}$ State Key Laboratory of Veterinary Etiological Biology, Key Laboratory of Veterinary Parasitology of Gansu Province, Lanzhou Veterinary Research Institute, CAAS, Lanzhou 730046, China

${ }^{2}$ Institute of Veterinary Medicine, Jiangsu Academy of Agricultural Sciences, Key Laboratory of Veterinary Biological Engineering and Technology, Ministry of Agriculture, National Center for Engineering Research of Veterinary Bio-products, Nanjing 210014, China

\section{Background}

Bovine viral diarrhea virus (BVDV) is important pathogen related to bovine gastrointestinal, respiratory and reproductive diseases and causes serious bovine viral diarrhea/mucosal disease (BVD-MD), which has spread worldwide [1-3]. BVDV is a single positive strand RNA virus and belongs to the genus Pestivirus of the Flaviviridae family. Infection commonly presents with immunosuppression, fever, diarrhea, gastroenteritis, erosion 
of digestive tract mucosa and reproductive disorders [4, 5]. More serious is that the immune dysfunction, immunosuppression and persistent infection (PI) caused by BVDV infection promotes the further spread of the virus and increasing morbidity and mortality in animals, which brings enormous economic losses to the cattle industry.

According to the 5'UTR sequence, three genotypes (BVDV-1, BVDV-2 and BVDV-3) were identified within BVDV strains. Among them, the highly pathogenic strain, BVDV-2, usually causes severe acute infection and hemorrhagic syndrome $[6,7]$. There are two biotypes in BVDV, cytopathogenic biotype (CP) and non-cytopathogenic biotype (NCP) [8]. Except for the difference in pathogenicity (CP BVDV causes cell vacuolation, shedding and necrosis, but NCP BVDV does not), the genomes of CP and NCP BVDV-2 show obvious differences in the NS2/3 coding area. A number of studies have indicated that no insertions with exogenous sequence in NS2/3 coding regions have been observed in the NCP BVDV, but an insertion, deletion or RNA recombination of exogenous sequences in the NS2/3 region was present in CP BVDV [9-11]. However, the novel CP BVDV-2 GS2018 strain isolated by our research group, is without inserted sequence in NS2/3 coding region, but showed significant cytopathic effect in MDBK cells, compared with other common CP BVDV.

The mechanisms of BVDV-host interaction and pathogenesis are complex. Successful evasion of the host immune system is the basis for persistent BVDV infection. Previous studies have indicated that BVDV infection induced host peripheral blood lymphopenia, a reduction of cell proliferation activity, damaged cell function and induced apoptosis [12-18]. Other data showed that CP BVDV infection induced type I interferon production, whereas NCP BVDV did not [19], demonstrating that NCP BVDV could avoid the innate immune response. The Npro of NCP BVDV is able to block the DNA-binding activity of interferon regulatory factor 3 (IRF-3) [20, $21]$, and is the main cause of immunosuppression and persistent infection. Varied levels of immune response were induced by different biotypes of BVDV, although it is generally recognized that the Th1 response was stimulated by CP BVDV infection while NCP BVDV stimulated the Th2 response [22]. All strains can cause severe depletion of $\mathrm{T}$ lymphocytes subgroup.

It has been reported that $\mathrm{CD} 4{ }^{+}$effector $\mathrm{T}$ cells differentiate into Th17 cells, which are involved in inflammatory responses and play key roles in the host defense against extracellular pathogens [23, 24]. IL17, the main effector molecule produced by Th17 cells, is a proinflammatory cytokine that binds with the heterodimer IL17RA/C receptor to activate NF- $\mathrm{kB}$, MAPKs, C/EBPs and other common signaling pathways, which play key roles in inducing inflammatory autoimmune diseases and host defense [24]. Studies have reported that viral infection with HBV and PRRSV could lead to up-regulated host expression of IL17, affecting virus replication and disease outcome conversely [25-27]. Here, we focus on the expression levels of immune-related cytokines, especially IL17A, in bovine PBLCs infected with GS2018, which provides theoretical support for further research on the mechanism of host immune response and pathogenesis induced by BVDV infection.

\section{Results \\ Identifying high-quality reads}

In order to obtain high quality reads from the bovine PBLCs for further analysis, the cDNA sequencing library was constructed and sequenced based on the BGISEQ-500 sequencing platform. A total of 296,230,298 raw reads (NC: 147,238,728; CP: 148,991,570) were generated. After eliminating the low-quality reads, the 269,475,890 clean reads (NC: 134,012,654, CP: $135,463,236)$ were obtained with the Q20 > 97\%, indicating high-quality sequencing data. For further analysis, the clean reads were mapped to the reference Bos taurus genome (GCF_000003205.7_Btau_5.0.1) using HISAT. Approximately $86.59 \%$ of the clean reads were successfully mapped to the Bos taurus genome and $84.32 \%$ of the clean reads were mapped uniquely to the Bos taurus genome (Table 1).

\section{Differential expressed genes analysis}

To investigate the mRNA expression profile of PBLCs after GS2018 infection, the DEseq2 method was used to detect the DEGs between the control (NC) and infection group (CP). As shown in Fig. 1, a total of 1052 genes were found to be significantly differentially expressed $(|\log 2 \mathrm{FC}| \geq 1$ and $\mathrm{Q}$ value $<0.05)$. Among them, 485 mRNAs were up-regulated and 567 mRNAs were downregulated in the GS2018 group in comparison with the control group (Fig. 1 and Additional file 1: Tables S1 and S2). Following the preliminary analysis of the mRNA expression profile, 19 cytokine genes were selected for further qPCR validation. Accordingly, these genes presented similar expression tendency with the RNA-Seq (Fig. 2), confirming the reliability of mRNA expression profiling.

\section{Functional annotation and PPI analysis of DEGs}

In order to investigate changes in the patterns and associated functions of DEGs in PBLCs infected by BVDV, GO annotation and KEGG enrichment analysis were performed on DEGs. The 1052 DEGs were annotated to 58 different GO terms, with the most annotated GO terms being cellular process $(\mathrm{CP})$, biological regulation 
Table 1 Statistics for sequence quality control and mapped data of samples

\begin{tabular}{llllllllll}
\hline & Raw reads & Low quality reads & Clean reads & $\begin{array}{l}\text { Clean } \\
\text { bases (Gb) }\end{array}$ & & Q20 (\%) & Q30 (\%) & $\begin{array}{l}\text { Clean reads } \\
\text { ratio (\%) }\end{array}$ & $\begin{array}{l}\text { Total } \\
\text { mapping } \\
\text { (\%) }\end{array}$ \\
& & & & & $\begin{array}{l}\text { Uniquely } \\
\text { Mapping } \\
\text { (\%) }\end{array}$ \\
\hline NC1 & $49,079,576$ & $4,145,670$ & $44,933,906$ & 6.74 & 97.23 & 89.75 & 91.55 & 87.45 & 85.36 \\
NC2 & $49,079,576$ & $4,265,738$ & $44,813,838$ & 6.72 & 97.39 & 90.28 & 91.31 & 87.54 & 85.47 \\
NC3 & $49,079,576$ & $4,814,666$ & $44,264,910$ & 6.64 & 97.5 & 90.59 & 90.19 & 86.42 & 84.16 \\
CP1 & $49,079,576$ & $3,606,904$ & $45,472,672$ & 6.82 & 97.38 & 90.17 & 92.65 & 86.89 & 84.12 \\
CP2 & $50,832,418$ & $6,211,330$ & $44,621,088$ & 6.69 & 97.53 & 90.72 & 87.78 & 86.47 & 82.48 \\
CP3 & $49,079,576$ & $3,710,100$ & $45,369,476$ & 6.81 & 97.41 & 90.25 & 92.44 & 84.98 & 84.3 \\
\hline
\end{tabular}

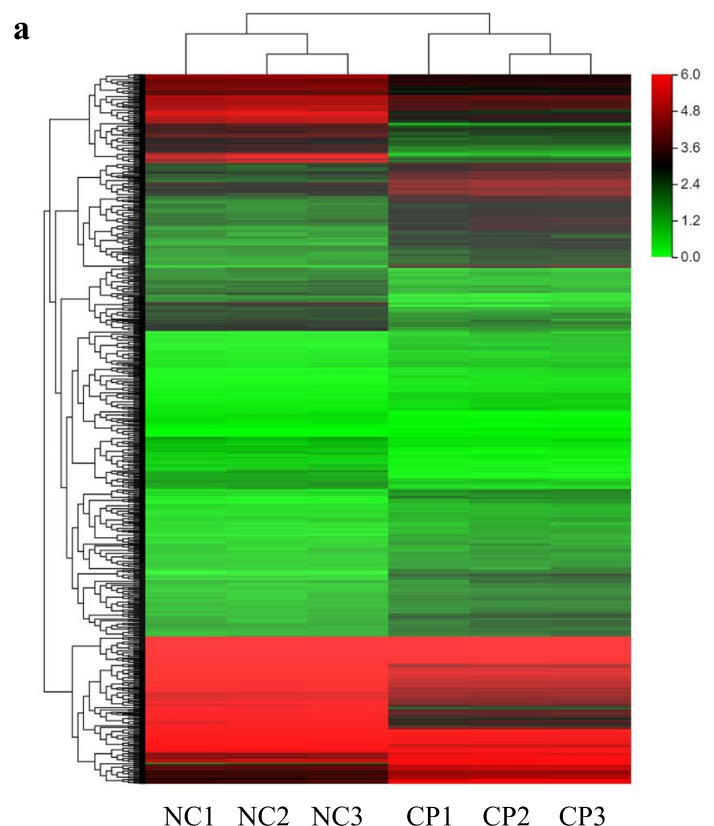

\section{b}

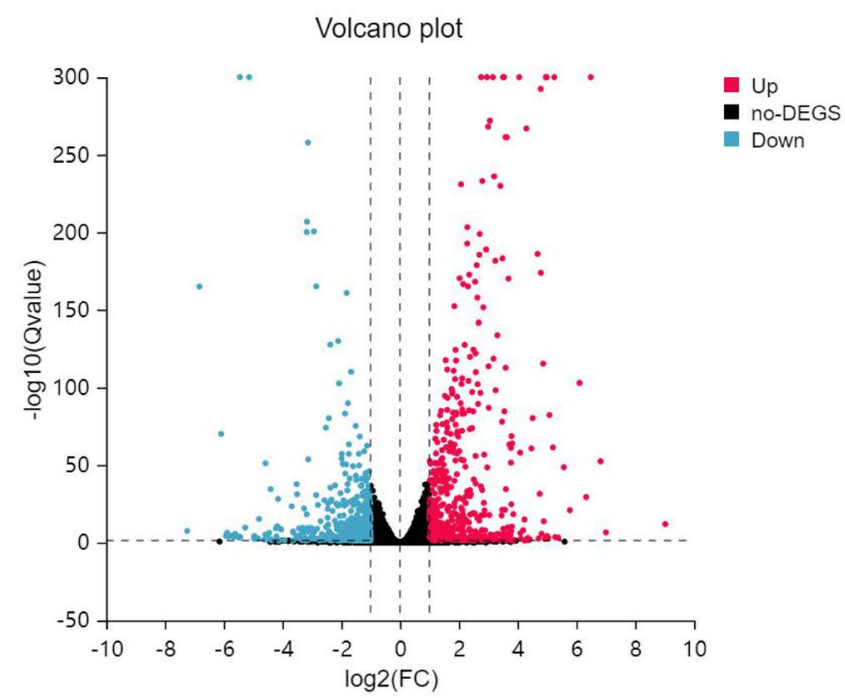

Fig. 1 Heat map and volcano plot of the DGEs between control and BVDV-2 infected group at $12 \mathrm{~h}$. a Heat map display the red represent the significantly up-regulated genes and the green represent the significantly down-regulated. $\mathbf{b}$ volcano plot show the red represent the significantly up-regulated genes and significantly down-regulated genes in blue

(BR), regulation of biological process (BP), response to stimulus, metabolic process (MP), signaling in BP categories, cell, cell part, organelle membrane, membrane part, organelle part in CC (cellular component) categories, and binding, catalytic activity, molecular function regulator and molecular transducer activity in MF (molecular function) categories (Fig. 3 and Additional file 2: Table S3). In addition, KEGG pathway enrichment analysis showed that these DEGs were significantly enriched in 16 pathways, including cytokine-cytokine receptor interaction, complement and coagulation cascades, NOD-like receptor, IL17, phosphatidylinositol-3-kinase (PI3K)/protein kinase $\mathrm{B}(\mathrm{Akt})$, mitogen-activated protein kinases
(MAPK) and tumor necrosis factor (TNF) signaling pathway (Fig. 4 and Additional file 3: Table S4). Among these pathways, cytokine-cytokine receptor interaction was the pathway with the highest number (61) of DEGs, 46 of which were up-regulated and 15 which were downregulated. Among these DEGs, CXCL10, IL17F, IFN$\gamma, I L 17 A$, and TNF were also significantly enriched in the IL17 signaling pathway (Fig. 5 and Additional file 4: Table S5).

Based on the data analysis results, we performed a protein-protein interaction analysis of the DEGs using the STRING database. The 4897 DEG pair interaction relationships were identified with significant values of 


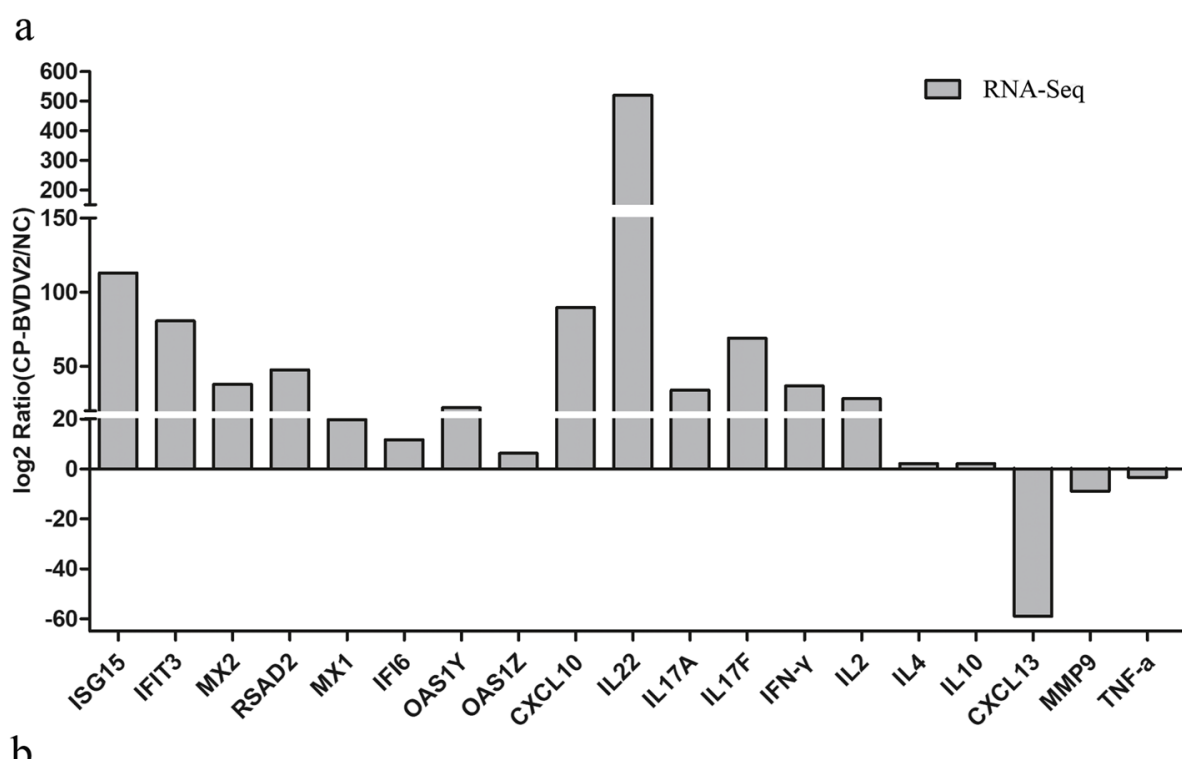

b

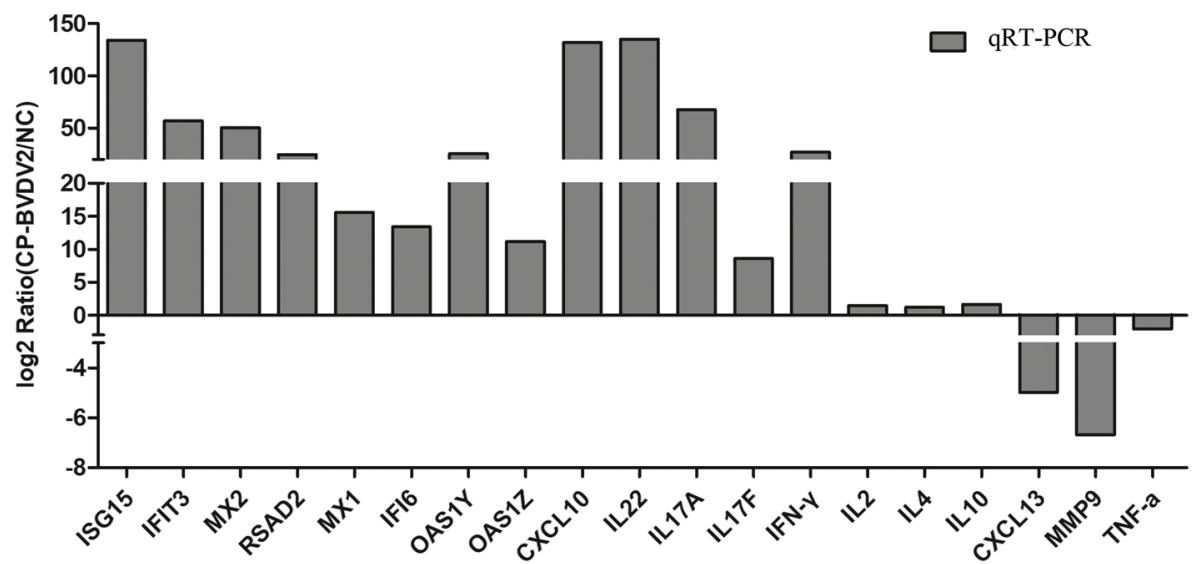

Fig. 2 Verification of the mRNA level of some DEGs by qRT-PCR. a The results of RNA-Seq. $\mathbf{b}$ The results of qRT-PCR. GAPDH was used as endogenous internal reference gene and the relative content was calculated according to the $2^{-\Delta \Delta C t}$ method

$p<0.05$. In order to understand better the interaction between various proteins, the candidate genes were selected for PPI network analysis using Cytoscape v3.6.1 software (Fig. 6 and Additional file 5: Table S6). The genes involved in IFN and IL signaling pathways, including IFN- $\gamma$, STAT1, CXCL10, IL17A, TNF- $\alpha$, $I S G 15$ and $I L 2$, played crucial roles in this network. In addition, these DEGs were related to the biological processes of defense response to virus, innate or adaptive immune responses, and regulation of signaling receptor activity. Moreover, IL17A, IL17F and IL22 are produced by Th17 cells and were significantly up-regulated, indicating that IL17 related cytokines might play important role in innate immune response and inflammatory response during BVDV infection.
Th17 cell differentiation was induced by GS2018 infection In order to determine whether Th17 cell differentiation was induced by GS2018 infection, the percentage of Th17 cells in PBLCs was determined by flow cytometry at $6 \mathrm{~h}, 12 \mathrm{~h}$ and $24 \mathrm{~h}$ post BVDV infection (pi). Interestingly, the Th17 cells were significantly increased $(22.72 \%$; 23.27 to $23.61 \%, p<0.01)$ in the GS2018 group compared with the control group $(18.58 \% ; 18.17$ to $19.31 \%)$ at $24 \mathrm{~h}$ pi. However, no significant difference was observed between the two groups at $6 \mathrm{~h}$ and $12 \mathrm{~h}(p>0.05)$ (Fig. 7). To further verify the effector molecules of Th17 cells, the expression of IL17A was detected by qPCR and western blotting in PBLCs at $12 \mathrm{~h}, 24 \mathrm{~h}, 48 \mathrm{~h}$ and $72 \mathrm{~h}$ after GS2018 infection. As shown in Fig. 8, at mRNA level, IL17A was 


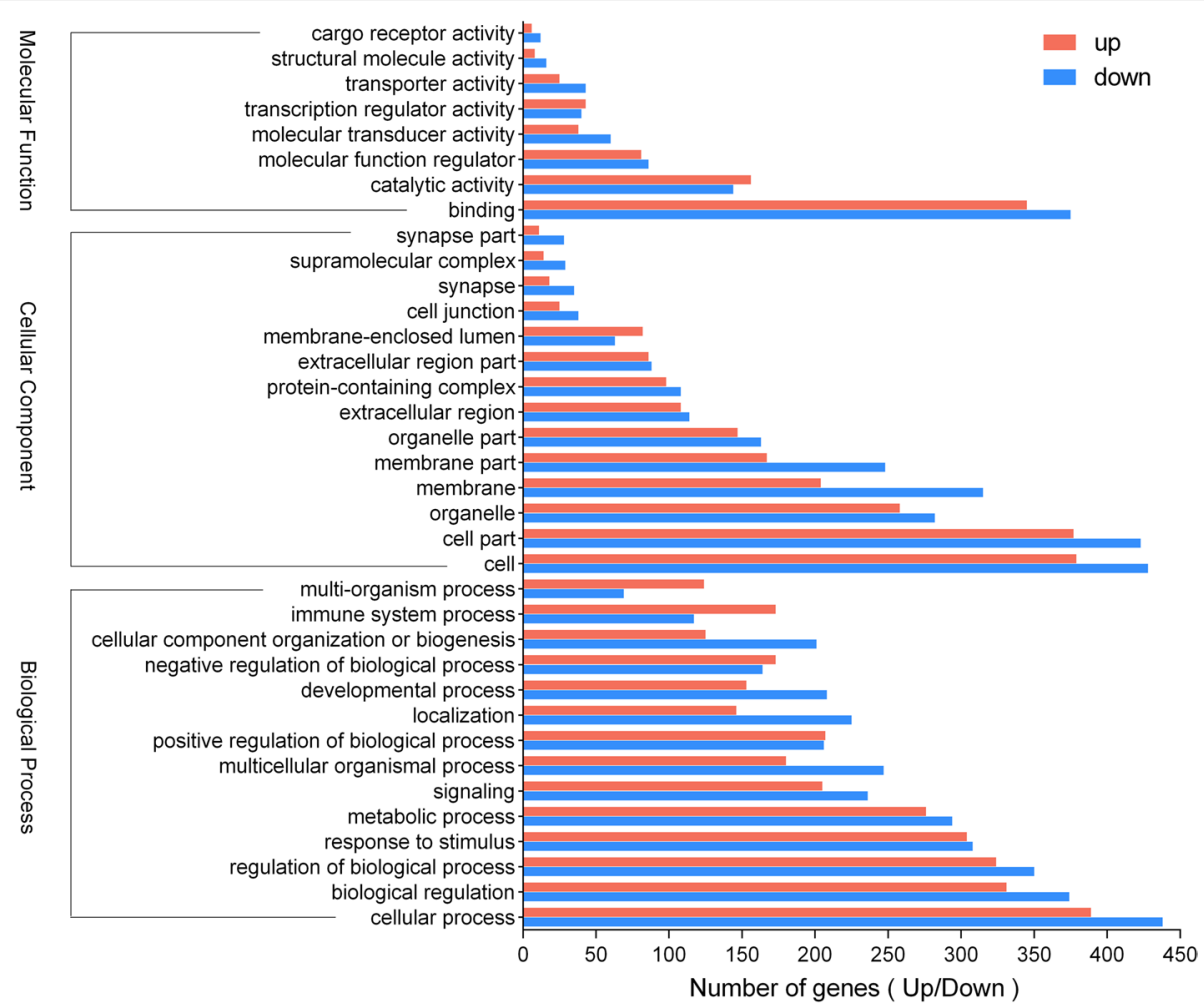

Fig. $3 \mathrm{GO}$ annotation for the DEGs between control and BVDV-2 infected bovine PBLCs. Horizontal axis represents the number of genes annotated on $\mathrm{GO}$ terms. Vertical axis represents the GO terms of BP, CC, and MF. Red represent the genes of up-regulated and the blue represent the down-regulated genes on different $\mathrm{GO}$ categories

\section{Significant Enriched Pathway Terms(top 20)}

RIG-I-like receptor signaling pathway

Toll-like receptor signaling pathway

FoxO signaling pathway

Proteasome

p53 signaling pathway

Phagosome $\square$

Chemokine signaling pathway

TNF signaling pathway

ECM-receptor interaction

MAPK signaling pathway

Osteoclast differentiation

PI3K-Akt signaling pathway

Hematopoietic cell lineage

Focal adhesion

Jak-STAT signaling pathway

C-type lectin receptor signaling pathway

IL-17 signaling pathway

NOD-like receptor signaling pathway

Complement and coagulation cascades

Cytokine-cytokine receptor interaction
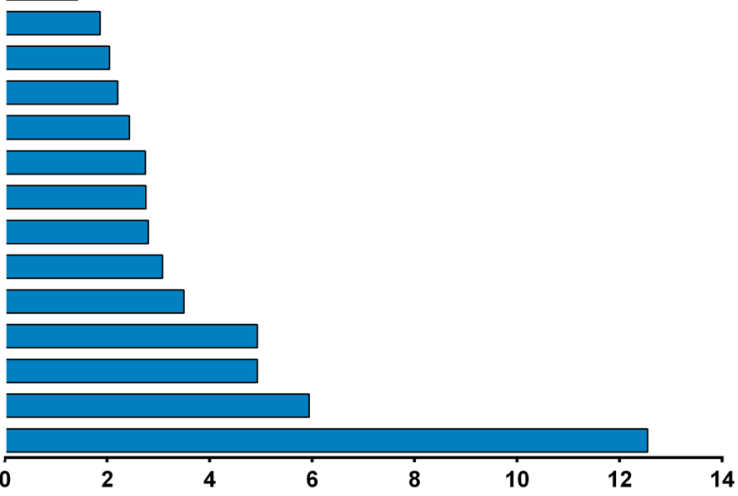

$-\log 10(Q-v a l u e)$

Fig. 4 KEGG pathway enriched pathway analysis of DEGs with top 20 enrichment scores [28] 


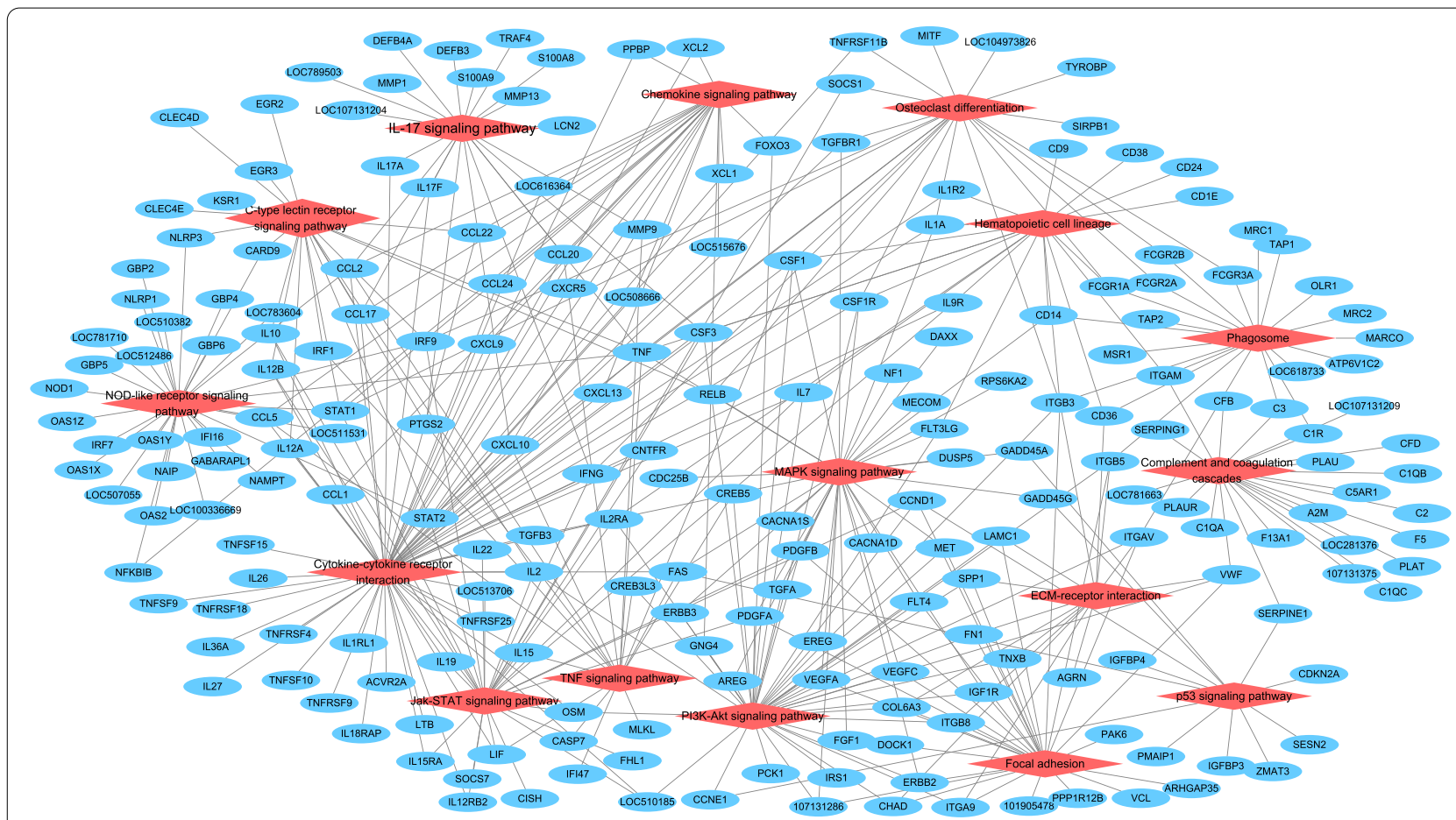

Fig. 5 KEGG interaction network analysis. The significantly differences 16 signaling pathways were represented in red and the DEGs in these pathways were represented in blue

up-regulated at least 10 folds at $48 \mathrm{~h}(p<0.01)$ and 80 folds at $72 \mathrm{~h}(p<0.01)$ compared with the uninfected group. The protein level of IL17A was up-regulated at 2 folds $(p<0.0001)$ at $48 \mathrm{~h}$ after GS2018 infection.

\section{IL17A inhibits GS2018 replication}

Based on the high expression levels of IL17A in PBLCs infected by GS2018, the PBLCs were used for a gain-offunction study. However, the transfection efficiencies of pEGFP-N1-IL17A in PBLCs were poor, so the PK15 cell line was used instead. The transfection efficiencies of pEGFP-N1-IL17A in PK15 were confirmed by fluorescence observation and qPCR (Fig. 9a-b). As expected, IL17A was localized in the cytoplasm with obvious punctate green fluorescence. We subsequently performed an overexpression assay of IL17A in PK15 cells, and then inoculated with GS2018 at a multiplicity of infection (MOI) of 1.0, which showed significant increase in IL17A $(p<0.01)$ at 24,48 and $72 \mathrm{~h}$ after infection (Fig. $9 \mathrm{~b}$ and Additional file 6: Fig. S1). Furthermore, the expression of the 5'UTR of GS2018 showed no obvious differences $(p>0.05)$ between the two groups at 24 and $48 \mathrm{~h}$ pi, but was down-regulated 1.6 fold at $72 \mathrm{~h}$ pi $(p<0.05$, Fig. 9c). Western blotting showed that the expression level of IL17A was significantly increased in the pEGFP-N1IL17A group at all times (Fig. 9d and Additional file 7:
Fig. S2), while the expression of GS2018 E2 protein was decreased 1.7 fold at $72 \mathrm{~h}$ pi compared with the control group ( $p<0.01$, Fig. 9d). Taken together, these results indicated that IL17A contributed to block in vitro replication of GS2018.

\section{Discussion}

BVD has been known for more than 70years and is one of the most important infectious diseases in stock farming due to its serious impact on animal husbandry worldwide. While some control and treatment measures have been improved, prevention and control of the disease remains a challenge due to the host immune function incapacitation, immune suppression and persistent infection. Therefore, elucidation of the mechanisms of host immunosuppression and immune function regulation caused by BVDV is urgent.

As a high-throughput sequencing technology, RNASeq has been used widely in different fields, providing an effective means to study the interaction between pathogens and hosts [29-31]. Although the transcriptomes of host cells inoculated with BVDV at the early stage of infection were sequenced [32-34], the mechanism responsible for the host immune function dysregulation caused by BVDV was not clear. In addition, accumulated evidence showed that different subtypes, 


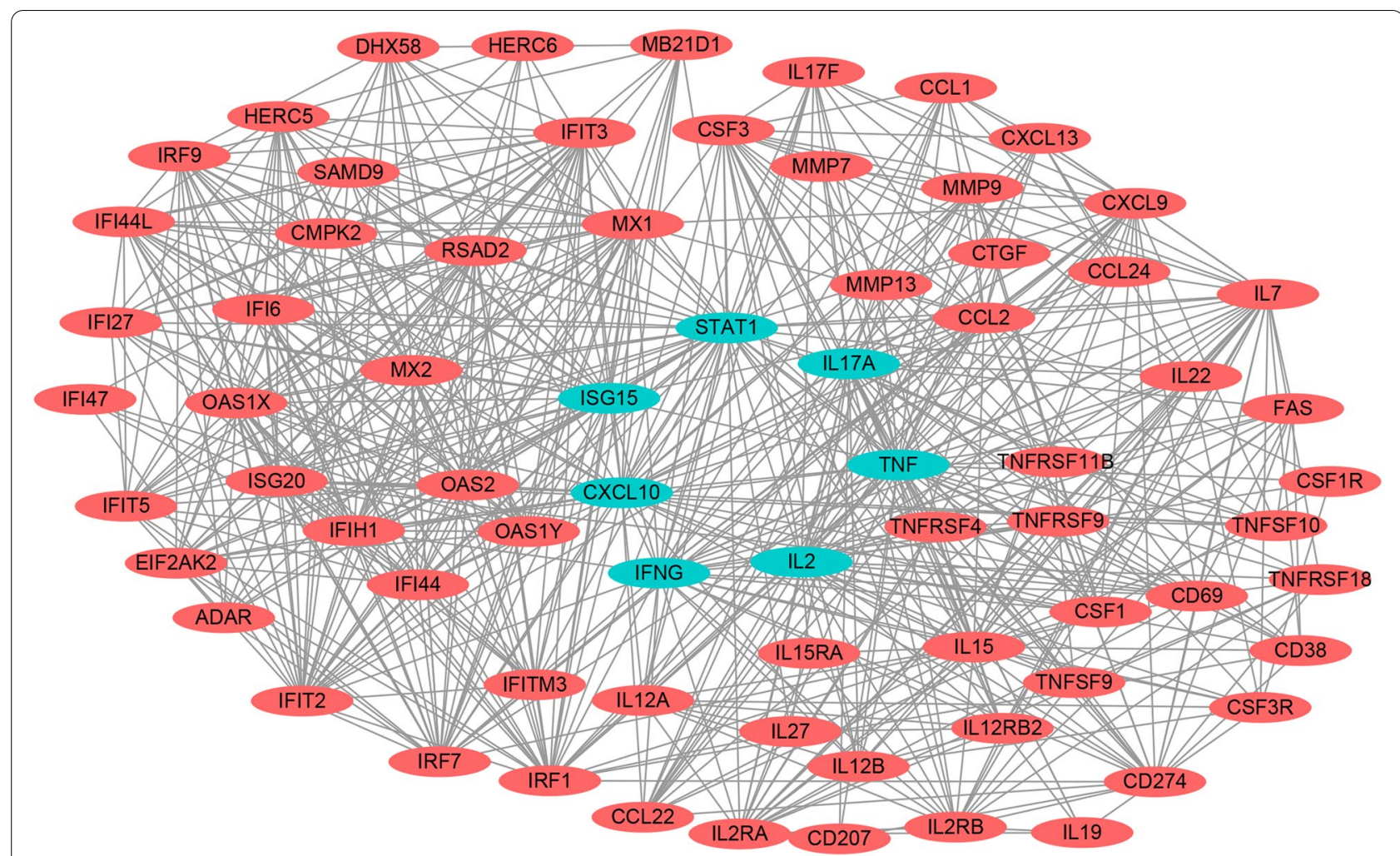

Fig. 6 Protein-protein interaction network analysis of DEGs between control and BVDV-2 infected bovine PBLCs. Parts of DEGs interaction relationships were identified with significant values of $p<0.05$ by using cytoscape_v3.6.1 software. The selected DEGs were represented in red circular and the green circular represents the genes with complex interaction network, the black solid line indicates the connection of each genes
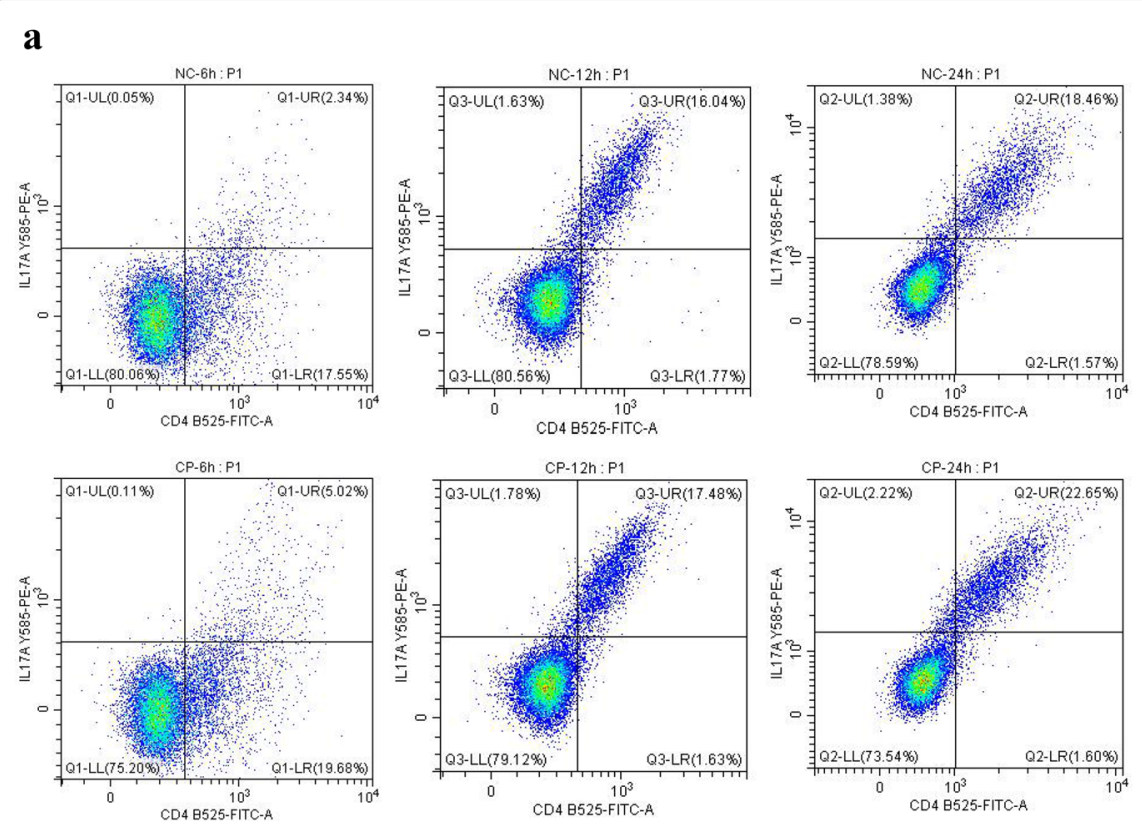

b

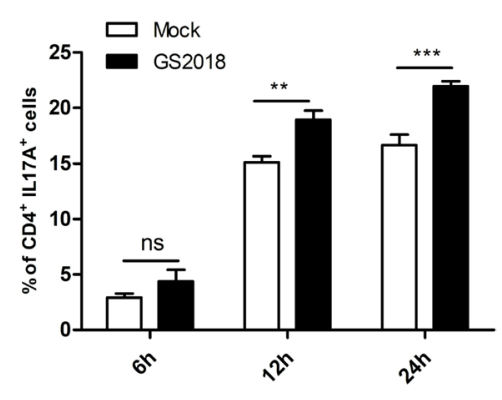

Fig. 7 Th17 cells differentiation was detected by flow cytometry. a Percentages of Th17 cells labeled by the CD4 and IL 17A monoclonal antibodies. b The bar chart shows the percentage of Th17 cells in control and GS2018 infection group at $6 \mathrm{~h}, 12 \mathrm{~h}$ and $24 \mathrm{~h}$, respectively. Data are representative of three independent experiments and presented as means \pm SDs. (ns, not significant; ${ }^{*} p<0.05$; ${ }^{* *} p<0.01$ ) 

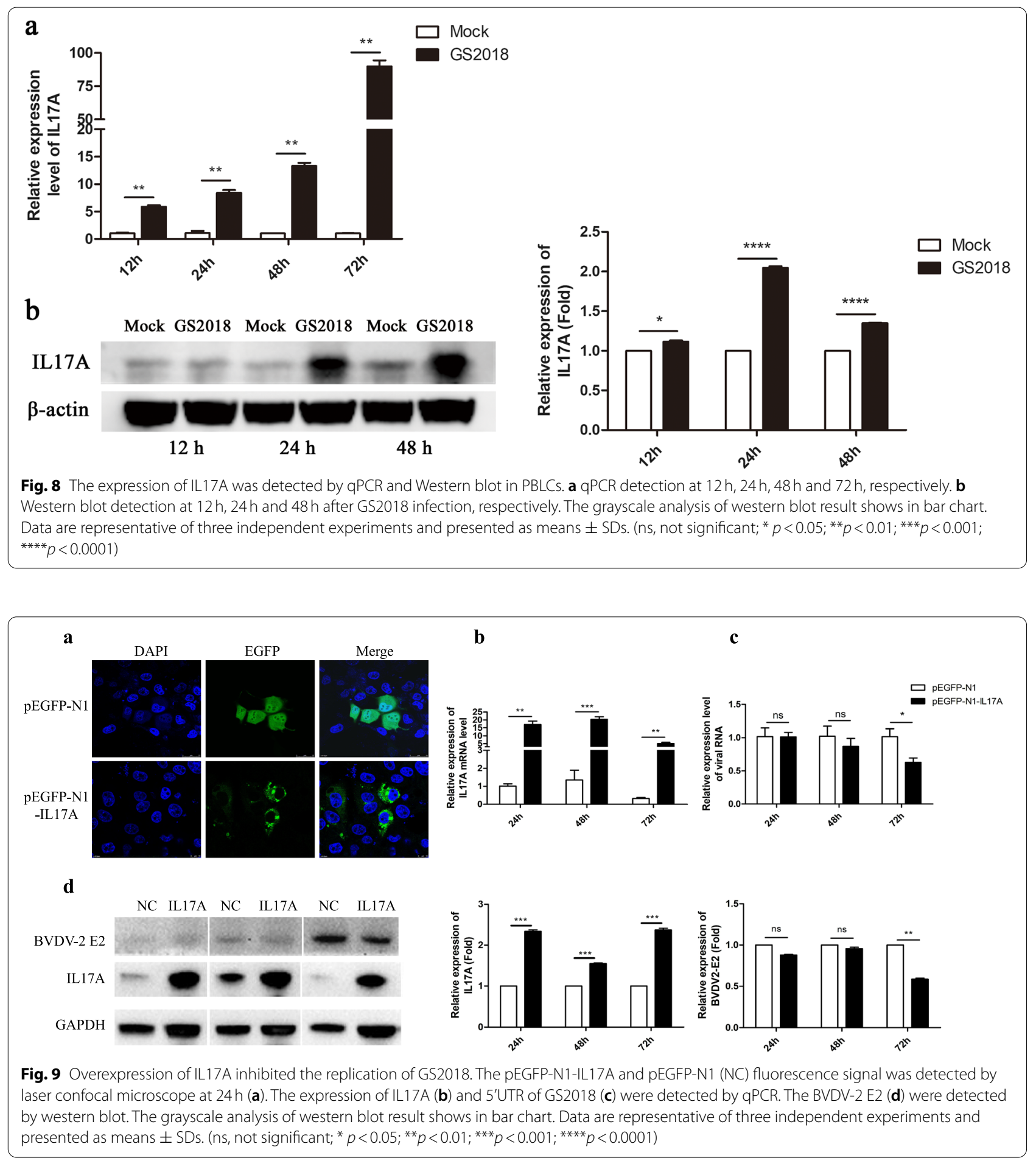

biotypes, and isolates of BVDV induced different levels of immune responses [35-39]. In this study, transcriptomic sequencing was performed for analysis of expression levels of genes in PBLCs at the early stage of infection with BVDV2 GS2018 strain, which provided basis data for understanding the response mechanism caused by early viral infection.

In this study, the transcriptomic analysis of bovine PBLCs indicated that most DEGs were involved in immune response, host defense, inflammatory reaction 
and cytokine-cytokine receptor interaction, which was consistent with previous research [32]. However, the role of the IL17 signaling pathway in the process was not frequently discussed The IFN and IL17 signaling pathway related genes, such as IFN- $\gamma$, STAT1, ISG15, CXCL10, $I L 17 A, T N F-\alpha$ and IL2, could interact with various cytokines, especially the significantly up-regulated interferon-stimulated genes (ISGs) and interferon-induced proteins forming the major interaction network. Previous research showed that myxovirus (influenza virus) resistance 1 (MX1) and ISG15 were up-regulated in the PBMCs from PI cattle which infected with BVDV-1 [40], suggesting that the $\mathrm{CP}$ and NCP BVDV could lead the production of ISGs to resist viral infection.

$\mathrm{CD} 4{ }^{+} \mathrm{T}$ helper (Th) cells can be divided into Th1, Th2, Th17, Foxp3 (+) T-regulating cells (Tregs), Th9 and Th22 cell subsets [41], which play key roles in maintaining the balance and stability of the immune system. Under the stimulation of some cytokines, such as IL23, Th17 cells differentiate and regulate inflammation responses by producing pro-inflammatory cytokines, chemokines and recruiting neutrophil infiltration [42, 43], which was independent of lineage. Interestingly, IL17A was induced sharply by GS2018 infection in this study. Furthermore, we also found that IL22, which together with IL17A and IL17F belongs to the Th17 effector molecules, was the most differentially expressed gene, at 521 folds higher than the NC group. Generally, the production of IL17A has been recognized as the marker of Th17 cell differentiation [44-47]. In order to determine whether the high levels of pro-inflammatory cytokines were caused by Th17 cell differentiation after BVDV infection, we measured the Th17 cell differentiation by flow cytometry and the production of IL17A by qRT-PCR and western blotting. The results confirmed the increase of Th17 cell differentiation and IL17 production after GS2018 infection. In turn, the high expression of IL17A blocked the replication of GS2018, which indicated that IL17A contributed to host antiviral immunity response.

In previous studies, Th17 cell production was dependent on transcription factor retinoid-associated orphan receptor $(\mathrm{ROR} \gamma \mathrm{t}$ ), which activates STAT3 under the stimulation of pro-inflammatory factors such as TGF$\beta$, IL1 $\beta$, IL-6, IL-21, and IL-23 [48-50]. However, in this study, the expression level of Th17 cell differentiation stimulating factors were not significantly changed, while its effector molecules such as IL17A, IL17F, IL22 were raised significantly. However, the mechanism of Th17 cell differentiation induced by GS2018 was still not clearly identified, and further research is needed. Recent studies confirmed that IL17 produced by Th17 cells could enhance the Th1 type immune response in mice infected by HSV-2, while in IL17-knockout mice the Th1 response was reduced and more susceptible to HSV-2 infection $[51,52]$. It is worthwhile to explore the effect of IL17A production on enhancing the Th1 immune response to resist BVDV.

Furthermore, a report about HCV found that the core and NS3 proteins could promote Th17 cell differentiation through stimulation of dendritic cells to secrete a large amount of IL6 and induce the production of IL17 from $\mathrm{CD} 4{ }^{+} \mathrm{T}$ cells, which play role in the autoimmune response of HCV patients [53]. Other research suggested that the nsp11 protein of PRRSV could up-regulate the expression of IL17A via activation of the IRAK1-PI3Kp38MAPK-C/EBP/CREB pathway, and that IL17 production was reduced and the pulmonary inflammation caused by HP-PRRSV infection was relieved by using PI3K inhibitor [26]. As discussed previously, induction of IL17 of host cells was beneficial for increasing production of pro-inflammatory cytokines, which may further promote pathogen clearance by the host. This effect was confirmed in our study, where the overexpression of IL17A significantly inhibited the replication of BVDV-2 GS2018. However, whether NS3 protein of GS2018 also has the potential to stimulate the production of IL17A remains to be investigated.

\section{Conclusion}

In this study, the immune regulatory mechanism of bovine PBLCs was explored using RNA-seq together with qRT-PCR, western blotting and flow cytometry to evaluate the effects of Th17 cell differentiation and IL17 production induced by CP BVDV-2 GS2018 infection. We found that the replication of GS2018 was inhibited by improving the expression of IL17A, providing a new basis for the study of BVDV-host interaction. In future studies, we will focus on the molecular mechanism of Th17 cell differentiation induced by GS2018, which will provide certain theoretical support for understanding the mechanism of host immune response induced by BVDV infection.

\section{Materials and methods Cells and virus}

Madin-Darby bovine kidney (MDBK, China Institute of Veterinary Drug Control, Beijing, China) were incubated in Dulbecco's Modified Eagle's medium-high glucose (DMEM, Hyclone Laboratories, Logan, UT, USA) containing $10 \%$ fetal bovine serum (Gibco FBS, Thermo Fisher Scientific, Waltham, MA, USA), $100 \mathrm{IU} / \mathrm{ml}$ penicillin and $100 \mathrm{IU} / \mathrm{ml}$ streptomycin at $37^{\circ} \mathrm{C}$ in $5 \% \mathrm{CO}_{2}$ humidified chamber. The CP BVDV-2 GS2018 strain 
(GenBank No.MN527354) was isolated from virus-contaminated commercial fetal bovine serum. The specific methodology has been described in our previous study [54].

\section{Virus titer assays}

Purified virus particles were diluted serially (1:10) in DMEM and inoculated into a 96-well culture plate containing $100 \mu \mathrm{l} /$ well of MDBK cells. The eight replicates in per-dilution and one negative control group were maintained at $37^{\circ} \mathrm{C}$ for $2 \mathrm{~h}$. Subsequently, the supernatants were aspirated, the cells were rinsed with phosphatebuffered saline (PBS), and fresh maintenance solution (DMEM medium containing 2\% FBS) was added to each well for $96 \mathrm{~h}$. On the fourth day post-infection, the number of wells with CPE in each dilution was determined and the $50 \%$ the tissue culture infective dose $\left(\mathrm{TCID}_{50}\right)$ of BVDV was calculated to be $1 \times 10^{6.2} \mathrm{TCID}_{50} / 100 \mu \mathrm{L}$ by the Reed-Muench method.

\section{Bovine PBLCs culture and virus infection}

Bovine peripheral blood was collected in EDTA anticoagulation tubes via carotid puncture. The PBLCs were separated from blood using a Bovine Peripheral Blood Lymphocyte Isolation Kit (TBD science, Tianjin, China) according to the manufacturer's instructions. RPMI-1640 medium (Gibco, Sunnyvale, CA, USA) supplemented with $10 \% \mathrm{FBS}$ and $1 \%$ penicillin-streptavidin was used to resuspend the PBLCs. After $4-5 \mathrm{~h}$ incubation at $37^{\circ} \mathrm{C}$, the PBLCs were harvested for counting and $3 \times 10^{6}$ cells were seeded into 6-well plates. Cells in each 6-well plate were set as either the CP or NC, with MOI of 0.1 GS2018 in the CP group and the same volume RPMI-1640 medium for the NC group. $12 \mathrm{~h} \mathrm{pi}$, the three replicate samples of each group were harvested for transcriptome sequencing (Beijing Genomics Institute, Shenzhen, PR, China) and qRT-PCR analysis.

\section{RNA extraction, library preparation and RNA sequencing (RNA-seq)}

Total RNA was extracted using TRIzol ${ }^{\circledR}$ Reagent (Invitrogen, Carlsbad, CA, USA) according to the manufacturer's instructions. The cDNA first strand was reverse transcribed based on the polyA tail method, and we switched template at the $5^{\prime}$ end of the RNA and amplified the full-length cDNA by PCR. The double stranded PCR products were purified using an Agencourt AMPure XPMedium kit (Beckman Coulter Life Sciences, San Jose, CA, USA). To obtain the single-stranded circle DNA (ssCir DNA) for library construction, the PCR products were heat denatured and circularized by the splint oligo sequence. An Agilent Technologies 2100 bioanalyzer
(Agilent RNA 6000 Nano Kit, Santa Clara, CA, USA) was used to assess the integrity and concentration of the ssCir DNA, and the library was amplified to create DNA nanoballs (DNB), containing more than 300 copies of each molecule.

The DNBs were dripped into the patterned nanoarray and single-end 50 base reads were generated by combinatorial probe-anchor synthesis (cPAS) sequencing. RIN/RQN values greater than 7.0 were used for mRNA sequencing. The raw data was filtered with SOAPnuke v1.5.2 (https://github.com/BGI-flexlab/SOAPnuke) to remove reads containing sequencing adapters, lowquality base ratio (reads with a mass value below 10 that accounted for more than $20 \%$ of the total base number of the reads) and unknown bases ('N' base) for obtaining clean reads. The clean reads were mapped to the reference bovine genome (GCF_000003205.7_Btau_5.0.1) using HISAT2 v2.0.4 (http://www.ccb.jhu.edu/software/ hisat/index.shtml). Ericscript v0.5.5 (http://ericscript. sourceforge.net/) and rMATS v3.2.5 (http://rnaseq-mats. sourceforge.net/) software were used for fusion genes and differential splicing genes (DSGs), respectively. Bowtie2 v2.2.5 (http://bowtie-bio.sourceforge.net/bowtie2) was used to align the clean reads to the gene set, which included the known and novel coding transcripts. Finally, the expression level of each gene was calculated using fragment per kilobase of exon model per million mapped reads (FPKM) by RSEM v1.2.12 (https://github.com/ deweylab/RSEM).

\section{Differential expression genes analysis and annotation}

DESeq2 with threshold of $|\log 2 \mathrm{FC}| \geq 1$ and $\mathrm{Q}$ value $<0.05$ were used to identify the differentially expressed genes (DEGs). To investigate the functions of DEGs, Gene Ontology (GO) functional annotation and Kyoko Encyclopedia of Genes and Genomes (KEGG) enrichment pathway analysis were performed on all DEGs. Among them, the categories molecular function (MF), cellular component $(\mathrm{CC})$ and biological process (BP) were analyzed in $\mathrm{GO}$ terms, with Q-value $<0.05$ defined as significantly enriched terms. The KEGG Pathway analysis performed to identify the biological functions of DEGs [28], with Q-value $<0.05$ considered as the significantly enriched pathways. Additionally, the protein-protein interaction network (PPI) relationship was analyzed using the String database (https://string-db.org/), and Cytoscape software was used to create the PPI network.

\section{Validation by qPCR}

Quantitative real-time polymerase chain reaction (qPCR) was performed to verify DEGs from transcriptomic sequencing. Nineteen genes including inflammatory 
factors were selected for qPCR verification, including 14 up-regulated genes (ISG15, IFIT3, MX2, RSAD2, MX1, IFI6, OAS1Y, OAS1Z, CXCL10, IL22, IL17A, IL17F, IFN$\gamma$ and $I L 2$ ), two genes with no differential expression (IL4 and IL10), and three down-regulated genes (CXCL13, $M M P 9, T N F-\alpha)$. First, the total RNA of all samples was extracted with TRIzol reagent according to the manufacturer's instructions. The cDNA first stand was synthesized by HiScript ${ }^{\circledR}$ III 1st Strand cDNA Synthesis Kit (+gDNA wiper). All-in-One qPCR Mix was used for qRT-PCR following the manufacturer's instructions. The $20 \mu \mathrm{l}$ reaction volume consisted of $10 \mu \mathrm{l} 2 \times$ all-in-one qPCR Mix, $0.4 \mu \mathrm{l}$ Rox Reference Dye, $0.4 \mu \mathrm{l}$ of upstream and downstream primers $(10 \mu \mathrm{mol} / \mathrm{l}), 2 \mu \mathrm{l}$ of cDNA template and $6.8 \mu \mathrm{l}$ of RNase-free water. The primers of the selected genes were designed by Sangon Biotech (Additional file 8: Table S7). The reaction was performed using an ABI 7500 Real-Time PCR System (Thermo Fisher, Waltham, MA, USA) with the following program parameters: $95^{\circ} \mathrm{C}$ for $10 \mathrm{~min}, 40 \mathrm{cycles}$ of $95^{\circ} \mathrm{C}$ for $10 \mathrm{~s}, 60^{\circ} \mathrm{C}$ for $1 \mathrm{~min}$. GAPDH was used as an endogenous internal reference gene, and each assay was performed in triplicate. The relative content was calculated according to the $2^{-\Delta \Delta C t}$ method.

\section{Recombinant plasmid pEGFP-N1-IL17A overexpression in PK15 cells}

The recombinant plasmid pEGFP-N1-IL17A was constructed with Hind III and BamH I restriction enzymes sites. The IL17 primers used in this study were as follows: sense primer, CCCAAGCTTATGGCTTCTATG AGAACTTCA, anti-sense primer, CGTGGATCCCGA GCCAAATGGCGG. Next, the pEGFP-N1-IL17A and the pEGFP-N1 vector were transfected into PK15 cells using INVI DNA RNA Transfection Reagent (Invigentech, Irvine, CA, USA) according to the manufacturer's protocol. After $24 \mathrm{~h}$, the cell nuclei were DAPI stained for $10 \mathrm{~min}$ at room temperature and identified by laser confocal fluorescent microscopy.

\section{Western blot analysis}

The proteins of PBLC were extracted by using RIPA lysis buffer containing $1 \%$ protease inhibitor. After determining protein concentration, a total of $30 \mu \mathrm{l}$ of samples were separated by $12 \%$ SDS-PAGE, transferred to a PVDF membrane and blocked with $7.5 \%$ skimmed milk at room temperature for $3 \mathrm{~h}$. The membrane was cropped horizontally according to the target protein and incubated overnight with rabbit anti-IL17A polyclonal antibody (1:1000 dilution, Abcam, Pleasanton, CA, USA) and rabbit anti-BVDV E2 polyclonal antibody (1:2000 dilution,
Bioss, Woburn, MA, USA) at $4{ }^{\circ} \mathrm{C}$. Anti-mouse IgG/HRP and goat anti-rabbit IgG/HRP antibody (1: 4000 dilution, Beyotime Biotechnology, Beijing, China) were used as secondary antibodies. Mouse anti-GAPDH and anti- $\beta$ actin monoclonal antibody (1:2000 dilution, Beyotime Biotechnology, Beijing, China) were used as an internal standard. The membrane was reacted with ultra-sensitive ECL luminescence reagent (Beyotime Biotechnology, Beijing, China) for color detection. All experiments were performed in triplicate. Grey-scale values of each blot were measured by Image J software, and the intensity of each band was normalized to the loading control GAPDH or $\beta$-actin.

\section{Flow cytometry data acquisition and analysis}

A total of $2 \times 10^{6}$ PBLCs were inoculated in a 12-well plate, with $\mathrm{CP}$ and $\mathrm{NC}$ groups. Flow cytometry was performed at $6 \mathrm{~h}, 12 \mathrm{~h}$ and $24 \mathrm{~h}$ pi. Briefly, the cells were resuspended in $100 \mu \mathrm{l}$ PBS, then filtered with $70 \mu \mathrm{m}$ mesh screen. The cells were fixed in $4 \%$ paraformaldehyde for $30 \mathrm{~min}$ on ice, centrifuged and resuspended in $100 \mu \mathrm{l} 1 \%$ Triton-100. The Th17 cells surface marker and IL17A were stained using FITC-labeled mouse anti-bovine CD4 (clone CC8, eBioscience, San Diego, CA, USA) antibody, PE-labeled rat anti-mouse IL17A (clone eBio17B7, eBioscience) antibody, and FITC/PE labeled mouse IgG2a kappa isotype control (clone eBM2a, eBioscience), respectively. Unstained and single-stained samples were used to adjust the fluorescence compensation level. All samples were stained on ice for $1 \mathrm{~h}$, washed with PBS, and resuspended in $500 \mu \mathrm{l}$ PBS. A flow cytometer (Beckman Coulter, Brea, CA, USA) was used to detect $\mathrm{CD}_{4}{ }^{+}$and IL17A ${ }^{+} \mathrm{T}$ cells and all data were analyzed using CytExpert software.

\section{Statistical analysis}

The Student's $t$-test was used to calculate statistically significant differences between the control and experimental groups. A $P$-value of less than 0.05 was considered to be statistically significant. All data were presented as mean \pm S.D. (standard deviation). ${ }^{*} p<0.05,{ }^{* *} p<0.01$, ${ }^{* * * *}$ $p<0.001$ and $* * * 0.0001$.

\section{Abbreviations}

BP: Biological process; BR: Biological regulation; BVD/MD: Bovine viral diarrhea/ mucosal disease; BVDV: Bovine viral diarrhea virus; CC: Cellular component; CP: Cytopathogenic biotype; DEGs: Differential expressed genes; DMEM: Dulbecco's Modified Eagle's medium-high glucose; FBS: Fetal bovine serum; FC: Fold change; GO: Gene Ontology; KEGG: Kyoko Encyclopedia of Genes and Genomes; MDBK: Madin-Darby bovine kidney; MF: Molecular function; MOI: Multiplicity of infection; MP: Metabolic process; NCP: Non-cytopathogenic biotype; PBLCs: Peripheral blood lymphocyte cells; PBS: Phosphate buffered saline; PI: Persistent infection; PPI: Protein-protein interaction; qRT-PCR: Quantitative real-time polymerase chain reaction; RNA-seq: RNA sequencing. 


\section{Supplementary Information}

The online version contains supplementary material available at https://doi. org/10.1186/s12864-021-08194-w.

Additional file 1: Table S1. The significantly differentially expressed mRNAs. Table S2. The volcano plot showed the distributions of mRNAs.

Additional file 2: Table S3. Go enrichment analysis of differentially expressed mRNAs.

Additional file 3: Table S4. KEGG pathway analysis of differentially expressed mRNAs.

Additional file 4: Table S5. KEGG interaction network analysis of differentially expressed mRNAs.

Additional file 5: Table S6. Protein-protein interaction network analysis of differentially expressed mRNAs.

Additional file 6: Figure S1. The expression of IL17A was detected by western blot in PBLCs at 12 h, 24h and $48 \mathrm{~h}$ after GS2018 infection, respectively. The above showing original blots of the targets protein. The band of IL17A $(a, b)$ and $\beta$-actin $(c, d)$ were cropped horizontally from the same membrane before hybridized with the antibody and then repeated exposures by using ultra-sensitive ECL luminescence reagent. For IL17A (17 kda and $14 \mathrm{kda}$ ) and $\beta$-actin (42 kda), membranes were cropped at $25 \mathrm{kda}, 35$ $\mathrm{kda}$ and $55 \mathrm{kda}$. Thereafter each membrane was processed for respective antibody incubation and detection as described in method section.

Additional file 7: Figure S2. Overexpression of IL17A inhibited the replication of GS2018. The Expression of IL17A and BVDV-2 E2 were detected by western blot in PBLCs at $24 \mathrm{~h}, 48 \mathrm{~h}$ and $72 \mathrm{~h}$ after GS2018 infection, respectively. The band of IL17A (a), BVDV-2 E2 (b, c) and GAPDH (d) protein were cropped horizontally from the same membrane before hybridized with the antibody, the image below shows the marker lanes as well as the complete and clearly visible edge of the gels. For BVDV-2 E2 (42 kda), GAPDH (37 kda) and pEGFP-N1-IL17A, membranes were cropped at 36 $\mathrm{kda}, 40 \mathrm{kda}$ and $60 \mathrm{kda}$. Thereafter each membrane was processed for respective antibody incubation and repeated exposures by using ultrasensitive ECL luminescence reagent.

Additional file 8: Table S7. Primers used for qRT-PCR.

\section{Acknowledgements}

The authors are sincerely thankful for the technical support provided by BGITech, Beijing Genomics Institute, Shenzhen, PR China.

\section{Authors' contributions}

XNL and LM designed the work and performed critical revision of the manuscript. YPL, TLL, GLC and LQW performed the experiments, analyzed the data, created the figures, and drafted the manuscript. AMG, ZL and LP collected blood samples and separated the PBLCs. All authors have read and approved the final manuscript.

\section{Funding}

This study was supported by State Key Laboratory of Veterinary Biotechnology Foundation (SKLVBF202106) and the National Natural Science Foundation of China (No. 31802196).

\section{Availability of data and materials}

The RNA sequencing data generated and analyzed during the present study have been submitted to NCBI project PRJNA683156 (https://www. ncbi.nlm.nih.gov/bioproject/PRJNA683156) with the accession number of SRR13318526 and SRR13318525.

\section{Declarations}

\section{Ethics approval and consent to participate}

The collection of bovine peripheral blood samples in this study was conducted under the guidance of experimental animal managers. All animal experiments were approved by the Animal Ethics Committee of the Lanzhou Veterinary Research Institute, Chinese Academy of Agricultural Sciences. All the experimental operations were accordance with the requirements of Laboratory animal-Guideline for Ethics review of animal welfare of the People's Republic of China (GB/T35892-2018) and the study complies with the ARRIVE guidelines (https://arriveguidelines.org).

\section{Consent for publication}

Not applicable.

\section{Competing interests}

The authors declare that no competing financial interests exist.

Received: 14 April 2021 Accepted: 17 November 2021

Published online: 07 December 2021

\section{References}

1. Gunn GJ, Saatkamp HW, Humphry RW, Stott AW. Assessing economic and social pressure for the control of bovine viral diarrhoea virus. Prev Vet Med. 2005;72(1-2):149-62.

2. Pinior $B$, Firth $C L$, Richter $V$, Lebl $K$, Trauffler M. A systematic review of financial and economic assessments of bovine viral diarrhea virus (BVDV) prevention and mitigation activities worldwide. Prev Vet Med. 2017;137(Pt A):77-92.

3. Seong G, Lee JS, Lee KH, Shin SU, Yoon JY, et al. Noncytopathic bovine viral diarrhea virus 2 impairs virus control in a mouse model. Arch Virol. 2016;161(2):395-403.

4. Lanyon SR, Hill Fl, Reichel MP, Brownlie J. Bovine viral diarrhoea: pathogenesis and diagnosis. Vet J. 2014;199(2):201-9.

5. Timurkan MO, Aydin H. Increased genetic diversity of BVDV strains circulating in eastern Anatolia, Turkey: first detection of BVDV-3 in Turkey. Trop Anim Health Prod. 2019:51(7):1953-61.

6. Darweesh MF, Rajput MK, Braun LJ, Ridpath JF, Neill JD, et al. Characterization of the cytopathic BVDV strains isolated from 13 mucosal disease cases arising in a cattle herd. Virus Res. 2015;195:141-7.

7. Yesilbag K, Alpay G, Becher P. Variability and global distribution of subgenotypes of bovine viral diarrhea virus. Viruses. 2017;9(6):128.

8. Decaro N, Lucente MS, Lanave G, Gargano P, Larocca V, et al. Evidence for circulation of bovine viral diarrhoea virus type $2 \mathrm{c}$ in ruminants in southern Italy. Transbound Emerg Dis. 2017;64(6):1935-44.

9. Sato A, Kameyama K, Nagai M, Tateishi K, Ohmori K, et al. Complete genome sequence of bovine viral diarrhea virus 2 Japanese reference and vaccine strain KZ-91CP. Genome Announc. 2015;3(1):e01573-14.

10. Vilcek S, Greiser-Wilke I, Nettleton P, Paton DJ. Cellular insertions in the NS2-3 genome region of cytopathic bovine viral diarrhoea virus (BVDV) isolates. Vet Microbiol. 2000;77(1-2):129-36.

11. Wang FI, Deng MC, Huang YL, Chang CY. Structures and functions of pestivirus glycoproteins: not simply surface matters. Viruses. 2015;7(7):3506-29.

12. Abdelsalam K, Rajput M, Elmowalid G, Sobraske J, Thakur N, et al. The effect of bovine viral diarrhea virus (BVDV) strains and the corresponding infected-macrophages' supernatant on macrophage inflammatory function and lymphocyte apoptosis. Viruses. 2020;12(7):701

13. Cardoso N, Franco-Mahecha OL, Czepluch W, Quintana ME, Malacari DA, et al. Bovine viral diarrhea virus infects monocyte-derived bovine dendritic cells by an E2-glycoprotein-mediated mechanism and transiently impairs antigen presentation. Viral Immunol. 2016;29(7):417-29.

14. Gamlen T, Richards KH, Mankouri J, Hudson L, McCauley J, et al. Expression of the NS3 protease of cytopathogenic bovine viral diarrhea virus results in the induction of apoptosis but does not block activation of the beta interferon promoter. J Gen Virol. 2010;91(Pt 1):133-44.

15. Hilbe M, Girao V, Bachofen C, Schweizer M, Zlinszky K, et al. Apoptosis in bovine viral diarrhea virus (BVDV)-induced mucosal disease lesions: a histological, immunohistological, and virological investigation. Vet Pathol. 2013;50(1):46-55.

16. Malacari DA, Pecora A, Perez Aguirreburualde MS, Cardoso NP, Odeon AC, et al. In vitro and in vivo characterization of a typical and a high pathogenic bovine viral diarrhea virus type II strains. Front Vet Sci. 2018;5:75.

17. Pedrera M, Gomez-Villamandos JC, Risalde MA, Molina V, Sanchez-Cordon PJ. Characterization of apoptosis pathways (intrinsic and extrinsic) in lymphoid tissues of calves inoculated with non-cytopathic bovine viral diarrhoea virus genotype-1. J Comp Pathol. 2012;146(1):30-9. 
18. Yamane D, Kato K, Tohya Y, Akashi H. The double-stranded RNA-induced apoptosis pathway is involved in the cytopathogenicity of cytopathogenic bovine viral diarrhea virus. J Gen Virol. 2006;87(Pt 10):2961-70.

19. Adler B, Adler H, Pfister H, Jungi TW, Peterhans E. Macrophages infected with cytopathic bovine viral diarrhea virus release a factor(s) capable of priming uninfected macrophages for activation-induced apoptosis. J Virol. 1997;71(4):3255-8.

20. Hansen TR, Smirnova NP, Webb BT, Bielefeldt-Ohmann H, Sacco RE, et al. Innate and adaptive immune responses to in utero infection with bovine viral diarrhea virus. Anim Health Res Rev. 2015;16(1):15-26.

21. Hilton L, Moganeradj K, Zhang G, Chen YH, Randall RE, et al. The NPro product of bovine viral diarrhea virus inhibits DNA binding by interferon regulatory factor 3 and targets it for proteasomal degradation. J Virol. 2006;80(23):11723-32.

22. Chase CC. The impact of BVDV infection on adaptive immunity. Biologicals. 2013;41(1):52-60.

23. Kaufmann U, Kahlfuss S, Yang J, Ivanova E, Koralov SB, et al. Calcium signaling controls pathogenic Th17 cell-mediated inflammation by regulating mitochondrial function. Cell Metab. 2019;29(5):1104-1118.e6.

24. Miossec P, Kolls JK. Targeting IL17 and TH17 cells in chronic inflammation. Nat Rev Drug Discov. 2012;11(10):763-76.

25. Cheng $X$, Shan YY, Luo YW. Dynamic change in Th17/regulatory T cell ratio in peripheral blood of patients with acute hepatitis $B$ and its significance. Zhonghua Gan Zang Bing Za Zhi. 2016;24(8):565-8.

26. Wang H, Du L, Liu F, Wei Z, Gao L, et al. Highly pathogenic porcine reproductive and respiratory syndrome virus induces Interleukin-17 production via activation of the IRAK1-PI3K-p38MAPK-C/EBPbeta/CREB pathways. J Virol. 2019;93(21):e01100-19.

27. Wang B, Zhao XP, Fan YC, Zhang JJ, Zhao J, et al. IL17A but not IL-22 suppresses the replication of hepatitis $B$ virus mediated by overexpression of MxA and OAS mRNA in the HepG2.2.15 cell line. Antivir Res. 2013;97(3):285-92.

28. Kanehisa M, Furumichi M, Sato Y, Ishiguro WM, Tanabe M. KEGG: integrating viruses and cellular organisms. Nucleic Acids Res. 2021;49(D1):D545-51.

29. Hicks JA, Yoo D, Liu HC. Transcriptional immune signatures of alveolar macrophages and the impact of the NLRP3 Inflammasome on porcine reproductive and respiratory syndrome virus (PRRSV) replication. Viruses. 2020;12(11):1299.

30. Kotliar D, Lin AE, Logue J, Hughes TK, Khoury NM, et al. Single-cell profiling of Ebola virus disease in vivo reveals viral and host dynamics. Cell. 2020;183(5):1383-1401.e19.

31. Liu W, Qiu X, Song C, Sun Y, Meng C, et al. Deep sequencing-based transcriptome profiling reveals avian interferon-stimulated genes and provides comprehensive insight into Newcastle disease virus-induced host responses. Viruses. 2018;10(4):162.

32. Li W, Mao L, Shu X, Liu R, Hao F, et al. Transcriptome analysis reveals differential immune related genes expression in bovine viral diarrhea virus-2 infected goat peripheral blood mononuclear cells (PBMCs). BMC Genomics. 2019;20(1):516.

33. Liu C, Liu Y, Liang L, Cui S, Zhang Y. RNA-Seq based transcriptome analysis during bovine viral diarrhoea virus (BVDV) infection. BMC Genomics. 2019;20(1):774.

34. Lopez BI, Santiago KG, Lee D, Ha S, Seo K. RNA sequencing (RNA-Seq) based transcriptome analysis in immune response of Holstein cattle to killed vaccine against bovine viral diarrhea virus type I. Animals (Basel). 2020;10(2):344

35. Hou L, Wilkerson M, Kapil S, Mosier D, Shuman W, et al. The effect of different bovine viral diarrhea virus genotypes and biotypes on the metabolic activity and activation status of bovine peripheral blood mononuclear cells. Viral Immunol. 1998;11(4):233-44.

36. Palomares RA, Brock KV, Walz PH. Differential expression of pro-inflammatory and anti-inflammatory cytokines during experimental infection with low or high virulence bovine viral diarrhea virus in beef calves. Vet Immunol Immunopathol. 2014;157(3-4):149-54.
37. Rajput MKS, Darweesh MF, Braun LJ, Mansour SMG, Chase CCL. Comparative humoral immune response against cytopathic or non-cytopathic bovine viral diarrhea virus infection. Res Vet Sci. 2020;129:109-16.

38. Riitho V, Larska M, Strong R, La Rocca SA, Locker N, et al. Comparative analysis of adaptive immune responses following experimental infections of cattle with bovine viral diarrhoea virus-1 and an Asiatic atypical ruminant pestivirus. Vaccine. 2018;36(30):4494-500.

39. Thakur N, Evans H, Abdelsalam K, Farr A, Rajput MKS, et al. Bovine viral diarrhea virus compromises Neutrophil's functions in strain dependent manner. Microb Pathog. 2020;149:104515.

40. Weng XG, Song QJ, Wu Q, Liu MC, Wang ML, et al. Genetic characterization of bovine viral diarrhea virus strains in Beijing, China and innate immune responses of peripheral blood mononuclear cells in persistently infected dairy cattle. J Vet Sci. 2015;16(4):491-500.

41. Muranski P, Restifo NP. Essentials of Th17 cell commitment and plasticity. Blood. 2013;121(13):2402-14

42. Harrington LE, Hatton RD, Mangan PR, Turner H, Murphy TL, et al. Interleukin 17-producing CD4+ effector T cells develop via a lineage distinct from the Thelper type 1 and 2 lineages. Nat Immunol. 2005;6(11):1123-32.

43. Park H, Li Z, Yang XO, Chang SH, Nurieva R, et al. A distinct lineage of CD4 $T$ cells regulates tissue inflammation by producing interleukin 17. Nat Immunol. 2005;6(11):1133-41.

44. Akiyama S, Asahina R, Ohta H, Tsukui T, Nishida H, et al. Th17 cells increase during maturation in peripheral blood of healthy dogs. Vet Immunol Immunopathol. 2019;209:17-21.

45. Lan YT, Wang ZL, Tian P, Gong XN, Fan YC, et al. Treg/Th17 imbalance and its clinical significance in patients with hepatitis B-associated liver cirrhosis. Diagn Pathol. 2019;14(1):114.

46. Liu MF, Wang CR. Increased Th17 cells in flow cytometer-sorted CD45ROpositive memory CD4 T cells from patients with systemic lupus erythematosus. Lupus Sci Med. 2014;1(1):e000062.

47. Rosado-Sanchez I, Herrero-Fernandez I, Tarancon-Diez L, Moreno S, Iribarren JA, et al. Increased frequencies of Th17 cells and IL17a-producing regulatory T-cells preceding the immunodiscordant response to antiretroviral treatment. J Inf Secur. 2018;76(1):86-92.

48. Chang H, Zhao F, Xie X, Liao Y, Song Y, et al. PPARalpha suppresses Th17 cell differentiation through IL-6/STAT3/RORgammat pathway in experimental autoimmune myocarditis. Exp Cell Res. 2019;375(1):22-30.

49. Korn T, Bettelli E, Oukka M, Kuchroo VK. IL 17 and Th17 cells. Annu Rev Immunol. 2009;27:485-517.

50. Mathur AN, Chang HC, Zisoulis DG, Stritesky GL, Yu Q, et al. Stat3 and Stat4 direct development of IL17-secreting Th cells. J Immunol. 2007;178(8):4901-7.

51. Anipindi VC, Bagri P, Roth K, Dizzell SE, Nguyen PV, et al. Estradiol enhances CD4+ T-cell anti-viral immunity by priming vaginal DCs to induce Th17 responses via an IL-1-dependent pathway. PLoS Pathog. 2016;12(5):e1005589.

52. Bagri P, Anipindi VC, Nguyen PV, Vitali D, Stampfli MR, et al. Novel role for Interleukin-17 in enhancing type 1 helper T cell immunity in the female genital tract following mucosal herpes simplex virus 2 vaccination. J Virol. 2017;91(23):e01234-17.

53. Tu Z, Hamalainen-Laanaya HK, Nishitani C, Kuroki Y, Crispe IN, et al. HCV core and NS3 proteins manipulate human blood-derived dendritic cell development and promote Th17 differentiation. Int Immunol. 2012;24(2):97-106.

54. Li YP, Wang LQ, Li HX, Liang PH, Cai XP, et al. Identification and complete genome sequencing analysis of a cytopathic BVDV-2 from commercial fetal bovine serum. Acta Vet Zootech Sin. 2020;51(02):320-8.

\section{Publisher's Note}

Springer Nature remains neutral with regard to jurisdictional claims in published maps and institutional affiliations. 